Check for updates

Cite this: RSC Adv., 2018, 8, 20655

\title{
Self-template construction of nanoporous carbon nanorods from a metal-organic framework for supercapacitor electrodes $\uparrow$
}

\author{
Ya-Wen Yang, ${ }^{a}$ Xuan-He Liu, (D) *a En-Peng Gao, ${ }^{a}$ Tian-Tian Feng, ${ }^{a}$ Wen-Jie Jiang, (D) ${ }^{b}$ \\ Jing Wu, ${ }^{a}$ HaO Jiang ${ }^{a}$ and Bing Sun (D) *a
}

\begin{abstract}
The morphologies and structures of nanostructured carbons generally influence their catalysis, electrochemical performance and adsorption properties. Metal-organic framework (MOF) nanocrystals usually have various morphologies, and can be considered as a template to construct nanostructured carbons with shaped nanocubes, nanorods, and hollow particles by thermal transformation. However, thermal carbonization of MOFs usually leads to collapse of MOF structures. Here, we report shape-preserved carbons (termed as CNRods) by thermal transformation of nickel catecholate framework (Ni-CAT) nanorods. Supercapacitors of CNRods treated at $800{ }^{\circ} \mathrm{C}$ were demonstrated to have enhanced performance due to their structural features that facilitate electron conduction and ion transport as well as abundant $\mathrm{O}$ content benefiting the wettability of the carbon materials. This may provide a potential way to explore novel carbon materials for supercapacitors with controllable morphologies and high capacitive performance.
\end{abstract}

Received 27th April 2018

Accepted 29th May 2018

DOI: $10.1039 / \mathrm{c} 8 \mathrm{ra03650k}$

rsc.li/rsc-advances

Inspired by their diverse crystalline morphologies and sizes,

\section{Introduction}

Nanostructured carbons, such as carbon nanotubes (CNTs), carbon nanoparticles, carbon capsules and graphene, have attracted much attention due to their applications in catalyst supports, electrochemical energy storage and gas storage. ${ }^{1-7}$ Generally, their electrochemical performance and adsorption properties are influenced by their morphologies and structures. Thus, scientists are committed to construct nanostructured carbons with geometrical control over them. Although several methods including chemical vapor decomposition (CVD), nanocasting, and electrical arc have been employed in preparing nanoporous carbons, ${ }^{2,4,5,8}$ simple, efficient and largescale production still remains a major challenge.

Porous metal-organic frameworks (MOFs) have attracted considerable attention due to their coordinated metal ion centers and robust nanoscale cavities and have been globally investigated in the fields of heterogeneous catalysis and gas separation and storage in the past decade. ${ }^{9-11}$ MOF nanocrystals with various morphologies, including nanocubes, nanorods and hollow particles have been shape-controlled by modulatorassisted methodology, microwave or ultrasonic method. ${ }^{12,13}$

${ }^{a}$ School of Science, China University of Geosciences (Beijing), Beijing 100083, China. E-mail: liuxh@cugb.edu.cn; sunbing@cugb.edu.cn; Tel: +8601082322758

${ }^{b}$ CAS Key Laboratory of Molecular Nanostructure and Nanotechnology, CAS Research/ Education Center for Excellence in Molecular Sciences, Institute of Chemistry, Chinese Academy of Sciences (CAS), Beijing 100190, China

$\dagger$ Electronic supplementary information (ESI) available: PXRD pattern, XPS spectra and electrochemical capacitive performance. See DOI: 10.1039/c8ra03650k
MOFs can be potentially considered as a template to construct nanostructured carbons with different morphologies by thermal transformation. ${ }^{14}$ However, thermal carbonization of MOFs usually results in decomposition of MOFs with the collapse of original morphologies. ${ }^{15-21}$ To date, self-template construction of nanoporous carbon with certain morphology still presents a significant challenge, although a few carbon materials in well-defined nanopolyhedra and nanorods have been obtained by thermal transformation of MOFs., ${ }^{1,22-24}$

Herein, we described one shape-preserved thermal transformation from the nanorod-shaped MOF to nanoporous carbon with the remaining morphology. The nanorod-shaped MOF, termed as nickel catecholate framework (Ni-CAT) was prepared by 2,3,6,7,10,11-hexahydroxytriphenylene $\left(\mathrm{H}_{12} \mathrm{C}_{18} \mathrm{O}_{6}\right.$, HHTP) linked with $\mathrm{Ni}(\mathrm{II})$ in an aqueous solution without any modulator. ${ }^{25,26}$ Nanorod-shaped nanoporous carbon with high specific surface area was obtained by shape-preserved thermal transformation of Ni-CAT. The nanoporous carbon nanorods were demonstrated to have good capacitive performance as supercapacitor electrode materials. In addition, the pyrolysis temperature, $\mathrm{Ni}$ and $\mathrm{O}$ contents involved in CNRods also affect the capacitive performance.

\section{Experimental}

\subsection{Synthesis of nanorod-shaped porous carbon}

Ni-CAT was synthesized through a convenient hydrothermal method. ${ }^{25}$ Typically, $40 \mathrm{~mL}$ aqueous solution with a mixture of $\mathrm{Ni}(\mathrm{OAc})_{2} \cdot 4 \mathrm{H}_{2} \mathrm{O}(100 \mathrm{mg})$ and HHTP $(70 \mathrm{mg})$ were transferred 
into a $100 \mathrm{~mL}$ Teflon-sealed autoclave. After being sealed, the mixed solution was heated to $85{ }^{\circ} \mathrm{C}$ for $12 \mathrm{~h}$. The nanorodshaped crystals were obtained by centrifugation and washed with deionized water and acetone. Nanorod-shaped nanoporous carbon was obtained by annealing Ni-CAT nanocrystals at a certain temperature $\left(700,800,900{ }^{\circ} \mathrm{C}\right)$ for $2 \mathrm{~h}$ under nitrogen atmosphere (Scheme 1). The heating rate was controlled as $5{ }^{\circ} \mathrm{C} \mathrm{min}^{-1}$. After cooling to room temperature, the product was collected and leached with $2 \mathrm{M} \mathrm{HCl}$ to remove big metallic Ni particles for characterization and electrochemical tests. The annealed products at $700{ }^{\circ} \mathrm{C}, 800{ }^{\circ} \mathrm{C}$, and $900{ }^{\circ} \mathrm{C}$ are termed as $\mathrm{CNRod}_{700}, \mathrm{CNRod}_{800}$, and $\mathrm{CNRod}_{900}$, respectively.

\subsection{Material characterization}

The morphologies of the Ni-CAT, $\mathrm{CNRod}_{700}, \mathrm{CNRod}_{800}$, and CNRod $_{900}$ were characterized on a JEM-2100F transmission electron microscopy (TEM) at an accelerating voltage of $200 \mathrm{kV}$. $\mathrm{X}$-ray diffraction (XRD) data were collected on a Rigaku D/Max2500 diffractometer at $40 \mathrm{kV}$ and $40 \mathrm{~mA}$ with $\mathrm{Cu} \mathrm{K} \alpha$ radiation $(\lambda=1.5416 \AA)$, ranging from $5^{\circ}$ to $80^{\circ}$ with a speed of $3^{\circ} \mathrm{min}^{-1}$ at ambient temperature. Raman spectra were recorded on a Thermo Scientific DXR Raman microscopy system equipped with a $533 \mathrm{~nm}$ laser. Nitrogen adsorption-desorption isotherms were carried out on Quantachrome Autosorb AS-1 Surface Area and Porosity Analyzer at liquid nitrogen temperature $\left(-196^{\circ} \mathrm{C}\right)$. The samples were degassed at $150{ }^{\circ} \mathrm{C}$ for at least $6 \mathrm{~h}$ under vacuum before measurements. X-ray photoelectron spectroscopy (XPS) spectra were obtained on a VG Scientific ESCALAB 250XI X-ray photoelectron spectrometer with a $200 \mathrm{~W}$ monochromatized Al-K $\alpha$ X-ray source $(1486.7 \mathrm{eV})$. The $500 \mu \mathrm{m}$ X-ray spot was used for XPS analysis. The base pressure in the analysis chamber was about $3 \times 10^{-10}$ mbar. Typically, the hydrocarbon C1s line at $284.8 \mathrm{eV}$ from adventitious carbon is used for binding energy referencing.

\subsection{Electrode preparation and electrochemical characterization}

The working electrodes were prepared by pressing the thoroughly mixed powder consisting of CNRod powder and polytetrafluorene-ethylene (PTFE) binder in a weight ratio of 95 : 5 onto nickel foam current collector $(0.5 \mathrm{~cm} \times 0.5 \mathrm{~cm})$, and then dried at $60{ }^{\circ} \mathrm{C}$ for $12 \mathrm{~h}$ under vacuum. All electrochemical tests were conducted in a three electrodes system (electrochemical capacitor) in $6.0 \mathrm{M} \mathrm{KOH}$ aqueous solution with $\mathrm{Hg}$ / $\mathrm{HgO}$ as the reference electrode and platinum foil $\left(1 \times 1 \mathrm{~cm}^{2}\right)$ as the counter electrode. All the electrochemical experiments were performed at ambient temperature (ca. $\left.25{ }^{\circ} \mathrm{C}\right)$. The

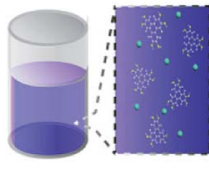

Precursors of Ni-CAT

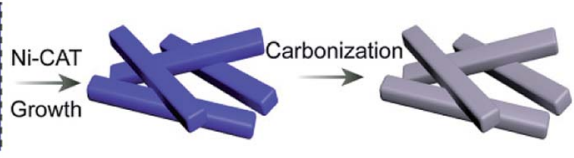

Ni-CAT
CNRod
Scheme 1 The schematic illustration of the synthetic procedure of $\mathrm{Ni}$ CAT and CNRods. prepared electrodes were soaked in the electrolyte solution overnight before electrochemical test. The cyclic voltammetry (CV) tests were performed on the electrochemical workstation (CHI 660E) in the potential window of -1.0 to $0 \mathrm{~V}$ at different sweep rates.

Chronopotentiometry measurements were also conducted at various current densities. Electrochemical impedance spectroscopy (EIS) of the capacitors was performed on an Autolab PGSTA302A electrochemical workstation in a frequency range of $0.01 \mathrm{~Hz}$ to $100 \mathrm{kHz}$ with perturbation amplitude of $5 \mathrm{mV}$.

\section{Results and discussion}

The Ni-CAT derived porous carbon materials were synthesized by annealing Ni-CAT at $700{ }^{\circ} \mathrm{C}, 800{ }^{\circ} \mathrm{C}$, and $900{ }^{\circ} \mathrm{C}$ under $\mathrm{N}_{2}$ atmosphere. The morphologies of as-prepared Ni-CAT and CNRods investigated by using transmission electron microscopy (TEM) and the TEM images are shown in Fig. 1. The NiCAT synthesized from HHTP and Ni(II) without any modulator-assisting shows the regular nanorod-like morphology (Fig. 1a). With the treatment at high pyrolysis temperatures $\left(700,800\right.$ and $\left.900{ }^{\circ} \mathrm{C}\right)$, the porous nanorod-shaped structures, termed as $\mathrm{CNRod}_{700}, \mathrm{CNRod}_{800}$, and $\mathrm{CNRod}_{900}$ are obtained (Fig. 1b-d). While metallic Ni particles form in the thermal treatment procedure, which can act as the catalyst for the partially graphitization of porous carbon during the thermolysis. The regular shape of Ni-CAT nanorod is well preserved as well as the size. The graphite layer fringes are clearly demonstrated according to the high-resolution TEM image (Fig. 1e). Apart from the few aggregated Ni particles, the Ni nanoparticles with the diameter of $10 \mathrm{~nm}$ are discretely encapsulated in highly carbonized CNRods.

The structures of as-prepared $\mathrm{CNRod}_{700}, \mathrm{CNRod}_{800}$, and CNRod $_{900}$ are investigated by using X-ray diffraction (XRD), Raman spectra and XPS spectra. The X-ray diffraction (XRD) pattern of Ni-CAT (ESI, Fig. S1 $\dagger$ ) indicates the well-defined crystalline structure. The intense diffraction peaks at $4.8^{\circ}$, $9.5^{\circ}, 14.2^{\circ}$, and $16.5^{\circ}$ show long-range order and well-developed
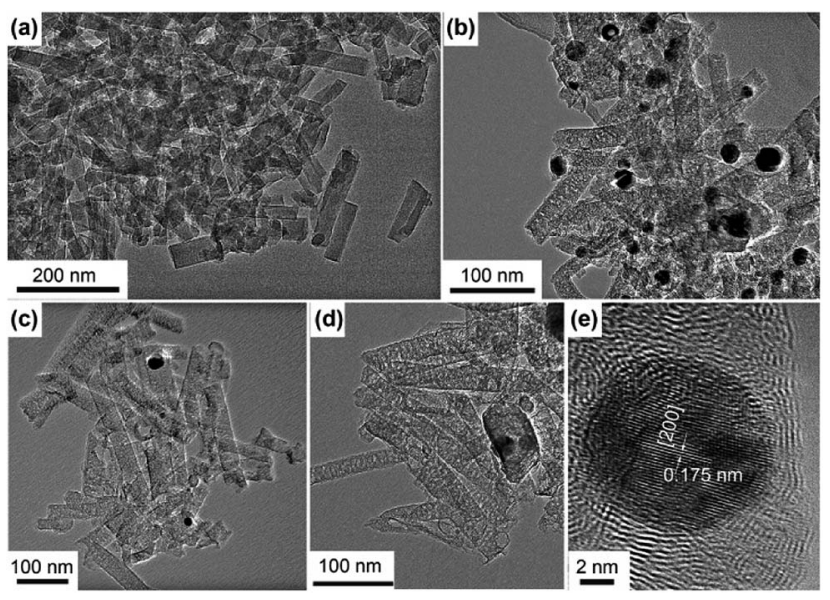

Fig. 1 TEM images of (a) nanorod-shaped Ni-CAT, (b) CNRod700, (c) CNRod $_{800}$ and (d) CNRod $_{900}$. (e) High resolution TEM image of CNRod $_{800}$. 
crystalline structure. After being carbonated at high temperature under $\mathrm{N}_{2}$ flow conditions, the XRD patterns of CNRods depict remarkable change compared to those of Ni-CAT (Fig. 2a). Three characteristic diffraction peaks appear at $44.5^{\circ}, 51.8^{\circ}$, and $76.4^{\circ}$, which can be indexed as Miller indices (111), (200), and (220) of face-centered cubic (fcc) metallic Ni (JCPDS no. 04-0850). The XRD pattern is corresponding with the high-resolution TEM image of metallic $\mathrm{Ni}$ nanoparticles (Fig. 1e). The weak and broad peak around $23^{\circ}$ can be attributed to the diffraction of amorphous carbon formed in the thermal treatment procedure. Along with the annealing temperature increased from 700 to $800{ }^{\circ} \mathrm{C}$, a characteristic diffraction peak with a sharp peak appears at $26.3^{\circ}$. Also, another weak diffraction peak emerges at $42.8^{\circ}$, which is implicit due to being overlapped by the (111) diffraction peak of Ni nanoparticles. These two peaks can be assigned to the (002) and (101) faces of formed graphitic carbon, respectively. Further raising the pyrolysis temperature to $900{ }^{\circ} \mathrm{C}$, the intensity of the (002) and (101) peaks enhanced, indicating the increased graphitization degree of CNRods.

The carbon structures of the as-prepared CNRods were further investigated by using Raman spectra. During the thermolysis in nitrogen atmosphere, the $\mathrm{G}$ band emerges at carbonization temperature higher than $600{ }^{\circ} \mathrm{C}$, which is attributed to the graphite in-plane vibrations with $\mathrm{E}_{2 \mathrm{~g}}$ symmetry. ${ }^{27,28}$ The D band arises from the disordered carbon structure or crystal defects. As shown in Fig. 2b, CNRods exhibit two characteristic Raman bands at $\sim 1350 \mathrm{~cm}^{-1}$ and $\sim 1580 \mathrm{~cm}^{-1}$, corresponding to $\mathrm{D}$ and $\mathrm{G}$ band, respectively. Additionally, a broad 2D band appears at $2680 \mathrm{~cm}^{-1}$ along with the carbonization temperature higher than $700{ }^{\circ} \mathrm{C}$, which is also an indication of highly ordered graphitic lattice. The relative intensity ratios of the $\mathrm{G}$ band to the $\mathrm{D}$ band demonstrate the graphitization degree. The $I_{\mathrm{G}} / I_{\mathrm{D}}$ values of CNRods are 1.18, 1.01 and 1.28 at 700,800 and $900{ }^{\circ} \mathrm{C}$, respectively. It indicates that CNRods contain both partially graphitized carbons and disordered carbons.
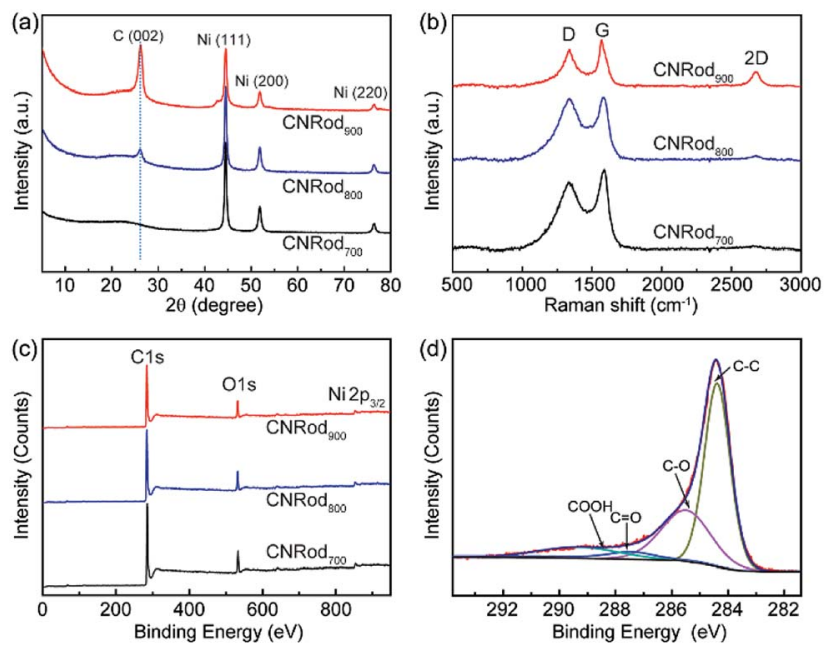

Fig. 2 (a) XRD patterns and (b) Raman spectra of the as-prepared CNRod $_{700}$, CNRod $_{800}$, and CNRod 900 . (c) Survey-scanned XPS spectrum and (d) high-resolution C1s spectrum of the CNRod 800 .
X-ray photoelectron spectroscopy (XPS) measurements were performed to investigate the chemical composition and element component of the as-prepared CNRods. The XPS survey spectra of CNRods reveal the presence of C1s, O1s and Ni2 $\mathrm{p}_{3 / 2}$ peaks without other signals (Fig. 2c). The C1s peaks for the Ni CAT-derived porous carbons (Fig. $2 \mathrm{~d}$ and S2, ESI $\dagger$ ) are centered at approximately $284.6 \mathrm{eV}$ (corresponding to $\mathrm{sp}^{2}$ graphitic carbon) and the shape of C1s peaks is slightly asymmetric due to the hetero-atom doping to carbon materials. The C1s spectrum of as-prepared CNRod $_{700}$ (Fig. S2a, ESI $\dagger$ ), CNRod $_{800}$ (Fig. 2d), and CNRod ${ }_{900}$ (Fig. S2b, ESI $\dagger$ ) can be deconvoluted as four bands at 284.4, 285.8, 287.5, and $289.0 \mathrm{eV}$, which represent the nonoxygenated ring $\mathrm{C}$, the $\mathrm{C}$ in $\mathrm{C}-\mathrm{O}, \mathrm{C}=\mathrm{O}$ and $-\mathrm{COOH}$ bonds, respectively. ${ }^{29}$ The stronger peak of $\mathrm{C}-\mathrm{C}$ indicated the better graphitization of graphene as the thermolysis temperature increases, being consistent with the sharper diffraction peaks in XRD patterns. The oxygen atoms in as-prepared $\mathrm{CNRod}_{700}, \mathrm{CNRod}_{800}$, and $\mathrm{CNRod}_{900}$ are approximately 11.0 at $\%, 8.8 \%$ and $6.5 \%$, respectively, which decreases with the increment of thermolysis temperature. The feature of oxygencontaining in CNRods would benefit the wettability of the carbon materials.

To get insight into the pore properties of the synthesized porous carbons, we measured the nitrogen adsorptiondesorption isotherms to compare specific Brunauer-EmmettTeller (BET) surface area of each sample and summarized total pore volume value. CNRods exhibits a combination of type II and type-IV isotherms (Fig. 3a), indicating the existence of both micropores and mesopores. The specific surface areas are 373, 367 and $375 \mathrm{~m}^{2} \mathrm{~g}^{-1}$ for $\mathrm{CNRod}_{700}, \mathrm{CNRod}_{800}$ and $\mathrm{CNRod}_{900}$, respectively. They are larger than that of Ni-CAT $\left(79.5 \mathrm{~m}^{2} \mathrm{~g}^{-1}\right) .{ }^{25}$ Fig. 3b displays DFT pore-size distribution curves of $\mathrm{CNRod}_{700}$, CNRod $_{800}$ and CNRod $_{900}$ calculated from the nitrogen desorption branches. The three kinds of CNRods depict the pore size centering in the region of mesopore and present close pore size distribution with a peak located at approximately $4.0 \mathrm{~nm}$ (ca. $3.87 \mathrm{~nm}, 3.93 \mathrm{~nm}$ and $3.86 \mathrm{~nm}$ for $\mathrm{CNRod}_{700}, \mathrm{CNRod}_{800}$ and CNRod $_{900}$, respectively), while the Ni-CAT only exhibits a pore size distribution at around $1.4 \mathrm{~nm} .^{25}$ The formation of mesopores and micropores can attributed to the gasification of the carbons during the carbonization procedure. With the thermal treatment temperature arising, no more pores are observed to be produced to lead to larger specific surface area. Thus, the NiCAT-derived CNRods possess the improved specific surface area and pore size in the carbonization procedure as well as remaining the fundamental porous structure and morphologies. The partially graphitized carbons and enhanced surface area can facilitate the electrochemically capacitive performance of CNRods.

Electrochemically capacitive performance of the as-prepared CNRods was investigated through cyclic voltammograms (CV) and galvanostatic charge-discharge tests by constructing a three-electrode symmetrical supercapacitor cell in $6.0 \mathrm{M} \mathrm{KOH}$ electrolyte. As shown in Fig. 4a, steady-state CV for the CNRods display regular rectangular-like shapes without redox peaks. The results indicate that CNRods show the capacitive behavior and there are no faradaic processes when cycled between the 


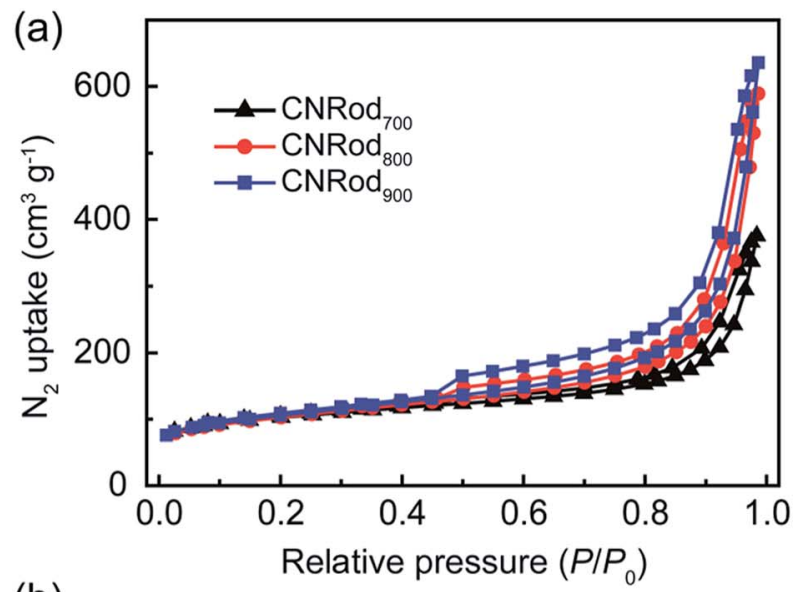

(b)

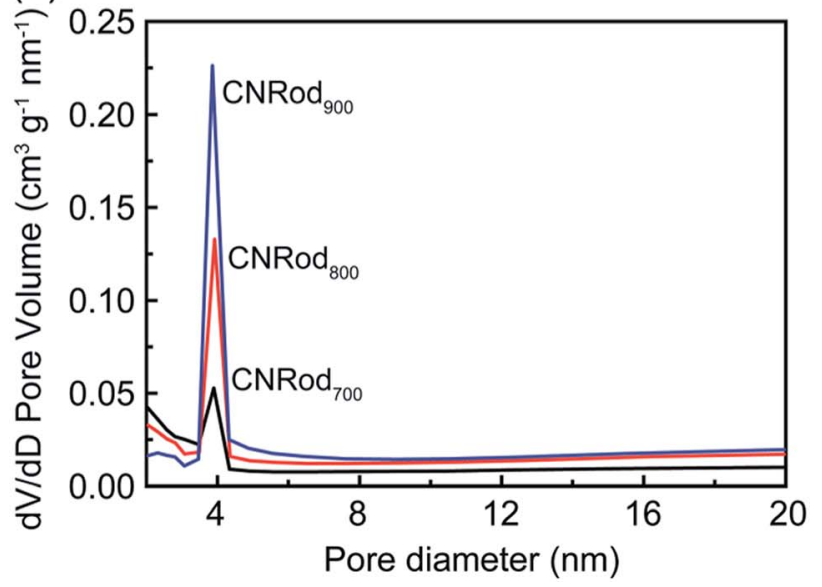

Fig. 3 (a) $\mathrm{N}_{2}$ adsorption-desorption isotherms curves and (b) poresize distribution curves of the as-prepared CNRod $_{700}, \mathrm{CNRod}_{800}$, and CNRod 900 .

potential range of $-1.0 \mathrm{~V}$ and $0 \mathrm{~V}$. The $\mathrm{CNRod}_{800}$-modified electrode has the widest $C V$ curve at the same scan rate of $30 \mathrm{mV} \mathrm{s}^{-1}$, which reveals the higher charge storage capacity than $\mathrm{CNRod}_{700}$ and $\mathrm{CNRod}_{900}$. The galvanostatic chargedischarge cycling tests display nearly triangular traces which are also indicative of capacitive behavior (as shown in Fig. 4b). The specific capacitances are calculated from galvanostatic discharge curves by using the equation: $C=I \Delta t / \Delta V$, where $I$ is the discharge current, $\Delta t$ is the total discharge time, and $\Delta V$ represented the discharging potentials window. The specific capacitances of the $\mathrm{CNRod}_{700}, \mathrm{CNRod}_{800}$ and $\mathrm{CNRod}_{900}$ modified electrodes are calculated as $67 \mathrm{~F} \mathrm{~g}^{-1}, 127 \mathrm{~F} \mathrm{~g}^{-1}$ and $98 \mathrm{~F} \mathrm{~g}^{-1}$, respectively, at a constant current density of $0.5 \mathrm{~A} \mathrm{~g}^{-1}$. CNRod 800 shows the best capacitive performance among the Ni-CATderived porous carbon materials, in the same range as the nano/mesoporous carbon materials. ${ }^{30}$ The normalized specific capacitance at $0.5 \mathrm{~A} \mathrm{~g}^{-1}$ is calculated as $34.6 \mu \mathrm{F} \mathrm{cm} \mathrm{cm}^{-2}$ which is larger than that of most porous carbon materials and some graphene or CNT-based materials. ${ }^{31}$ The energy density and power density of $\mathrm{CNRod}_{800}$-based electrochemical capacitor at $0.5 \mathrm{~A} \mathrm{~g}^{-1}$ are calculated as $4.4 \mathrm{~W} \mathrm{~h} \mathrm{~kg}^{-1}$ and $62.4 \mathrm{~W} \mathrm{~h} \mathrm{~kg}^{-1}$. Fig. 4c presents the $\mathrm{CV}$ for $\mathrm{CNRod}_{800}$ modified electrode at different scan rates ranging from $5 \mathrm{mV} \mathrm{s}^{-1}$ to $100 \mathrm{mV} \mathrm{s}^{-1}$. With
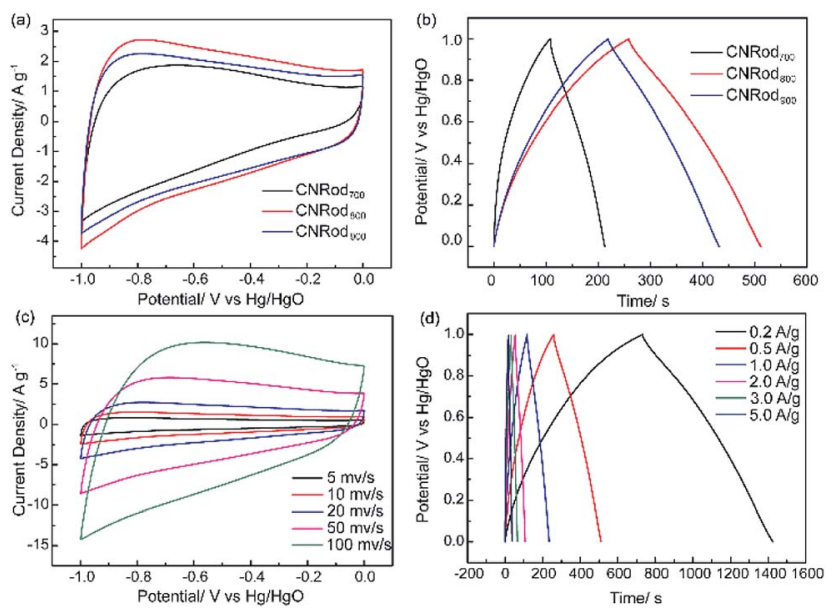

Fig. 4 Applications of carbon nanorods for electrochemical energy storage. (a) CV curves of the as-prepared CNRod $_{700}, \mathrm{CNRod}_{800}$ and CNRod $_{900}$ modified electrodes measured at $30 \mathrm{mV} \mathrm{s}^{-1}$ in $6.0 \mathrm{M} \mathrm{KOH}$ electrolyte. (b) Galvanostatic charge-discharge curves of the as-

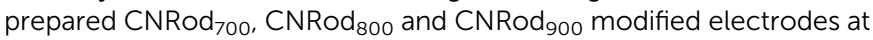
a constant current density of $0.5 \mathrm{~A} \mathrm{~g}^{-1}$. (c) CV curves of CNRod $800^{-}$ modified electrode measured at different scan rates in $6.0 \mathrm{M} \mathrm{KOH}$ electrolyte. (d) Galvanostatic charge-discharge curves of $\mathrm{CNRod}_{800}$ at different current densities.

the increment of scanning rate, the specific current is also gradually increased. The $C V$ curves of CNRod $_{800}$ modified electrode still show nearly rectangular shapes in the chosen potential range as at low scan rate when the scan rate reaches to $100 \mathrm{mV} \mathrm{s}^{-1}$, indicating its desirable fast charge-discharge property and well supercapacitor behavior. As shown in Fig. 4d, the galvanostatic charge-discharge curves of $\mathrm{CNRod}_{800}$ modified electrode at different current densities demonstrate only 17.4\% decay in specific capacitance at high current density of $5.0 \mathrm{~A} \mathrm{~g}^{-1}$ compared to that at $0.5 \mathrm{~A} \mathrm{~g}^{-1}$. In addition, the cycling

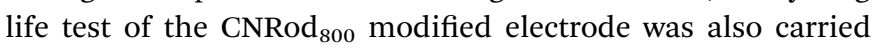
out. The capacitance still remains $100 \%$ after 2000 cycles at current density of $5.0 \mathrm{~A} \mathrm{~g}^{-1}$, indicating the good recycling

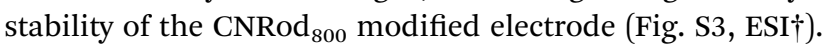

To understand the supercapacitor behavior of the three kinds of CNRods, electrochemical impedance spectroscopy (EIS) studies were carried out in $6.0 \mathrm{M} \mathrm{KOH}$ electrolyte in the frequency range from $100 \mathrm{kHz}$ to $0.01 \mathrm{~Hz}$ with the perturbation potential of $5 \mathrm{mV}$ (Fig. 5). In the Nyquist plots, the semicircles for CNRod materials in the high frequency ranges are very small indicating a small charge-transfer resistance $\left(R_{\mathrm{ct}}\right)$. The equivalent series resistance (ESR) of the Nyquist plots is defined as the intercept of plotting curves to the real axis in the high frequency range, and indicates the combined series resistance from the electrolyte, the electrodes, and electrical contacts. ${ }^{32}$ A small ESR value leads to better performances of energy-storage devices and to facilitate faster electron charge transport. The ESR of $\mathrm{CNRod}_{700}, \mathrm{CNRod}_{800}$, and $\mathrm{CNRod}_{900}$ is $0.698,0.300$ and $0.320 \Omega$, respectively. These results indicate that the higher graphitization in $\mathrm{CNRod}_{800}$ and $\mathrm{CNRod}_{900}$ endow them with faster electron transport rate, which is an essential factor to improve the capacitive performance of porous carbon materials. 


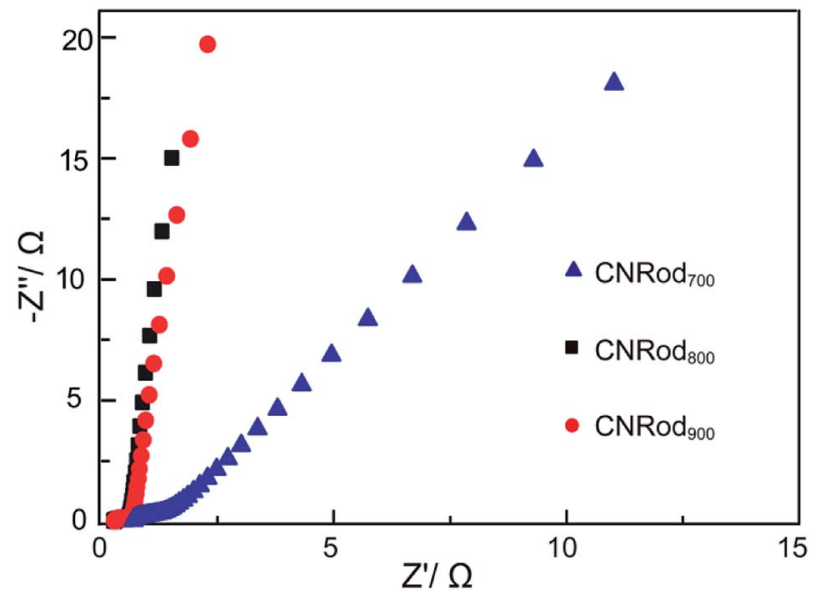

Fig. 5 Nyquist plots of the as-prepared CNRod $_{700}$, CNRod $_{800}$, and CNRodgoo modified electrodes.

In the low-frequency region, both $\mathrm{CNRod}_{800}$ and $\mathrm{CNRod}_{900}$ reveals straight and nearly vertical lines, indicating the typical characteristic of capacitive behavior, while CNRod ${ }_{700}$ exhibits a low slope linear curve. Particularly, CNRod ${ }_{800}$ shows the largest slope rate indicating the best capacitive behavior. The Warburg transition for $\mathrm{CNRod}_{700}, \mathrm{CNRod}_{800}$, and $\mathrm{CNRod}_{900}$ at the knee frequency is approximately $49.4 \mathrm{~Hz}, 15.8 \mathrm{~Hz}$, and $25.1 \mathrm{~Hz}$, respectively. The $45^{\circ}$ Warburg region in the intermediate frequency of the Nyquist plot reflects faster ions transport at the electrode/electrolyte interface of the porous CNRods. ${ }^{33}$ The shorter Warburg length indicates the shorter ion-diffusion path implying the faster ion kinetics. The Warburg length for CNRods are in the order of CNRod $_{800}<$ CNRod $_{900}<$ CNRod $_{700}$. The lower Warburg transition frequency and the shorter Warburg length may benefit the improved capacitive performance of $\mathrm{CNRod}_{800}$ modified electrodes compared to that of $\mathrm{CNRod}_{900}$ and $\mathrm{CNRod}_{700}$.

Typically, the capacitive performance is dependent on the carbonization temperature. The higher thermolysis temperature, the more excellent capacitive performance. While in this work, the $\mathrm{CNRod}_{800}$ modified electrode shows the highest specific capacitance slighter higher compared to CNRod $_{900}$ modified electrode as shown in the galvanostatic chargedischarge cycling tests (Fig. 4b). Given the similar specific BET surface area and total pore volume values by different thermal treatment temperature shown in nitrogen adsorption-desorption isotherms, $\mathrm{Ni}$ and $\mathrm{O}$ element composition of CNRods and the supercapacitor behavior was investigated by XPS and electrochemical characterization. The supercapacitor behavior of CNRod $_{800}$ modified electrodes with different $\mathrm{Ni}$ element composition was shown in Fig. S4 (ESI $\dagger$ ). The $\mathrm{CNRod}_{800}$ modified electrodes involved 0.22 at $\%, 0.42$ at $\%$ and 0.73 at $\% \mathrm{Ni}$ content exhibit similar capacitance, which indicates that $\mathrm{Ni}$ nanoparticles existing in $\mathrm{CNRod}_{800}$ may also have rare effect on capacitive behavior of CNRod ${ }_{800}$ modified electrodes. While, the more plentiful $\mathrm{O}$ contents in $\mathrm{CNRod}_{800}$ modified electrodes compared to that of $\mathrm{CNRod}_{900}$ may benefit the wettability of the carbon materials and enhanced the capacitive performance.

Based on the EIS results, the smaller ESR value and shorter

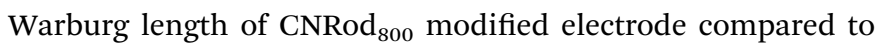

CNRod $_{900}$ indicate the faster electron charge transport and faster ions transport in $\mathrm{CNRod}_{800}$ material, implying the more enhanced capacitive performance. We think the best capacitive performance of $\mathrm{CNRod}_{800}$ may be ascribed to its suitable ion and electron transport as well as the plentiful oxygencontaining functional group being favorable to enhance the wettability of CNRod $_{800}$.

\section{Conclusions}

In summary, the shape-preserved thermal transformation from the nanorod-shaped MOF to nanoporous carbon with the same morphology was demonstrated in this work. The nanorodshaped nanoporous carbon termed as CNRods were demonstrated to have good performance for supercapacitor electrodes. Important factors of pyrolysis temperatures, $\mathrm{Ni}$ and $\mathrm{O}$ contents involved in CNRods affecting supercapacitor behavior were investigated. The capability of supercapacitors by CNRod $_{800}$ modified electrodes were demonstrated to be enhanced for their features of facilitating electron conduction and ion transport coupled with plentiful $\mathrm{O}$ contents benefiting the wettability of the carbon materials.

\section{Conflicts of interest}

There are no conflicts to declare.

\section{Acknowledgements}

This work was supported by National Natural Science Foundation of China (2160030921) and Fundamental Research Funds for the Central Universities (2652017143).

\section{Notes and references}

1 P. Pachfule, D. Shinde, M. Majumder and Q. Xu, Nat. Chem., 2016, 8, 718.

2 C. Journet, W. K. Maser, P. Bernier, A. Loiseau, M. L. de la Chapelle, S. Lefrant, P. Deniard, R. Lee and J. E. Fischer, Nature, 1997, 388, 756.

3 D. Higgins, P. Zamani, A. Yu and Z. Chen, Energy Environ. Sci., 2016, 9, 357-390.

4 Q.-H. Yang, P.-X. Hou, M. Unno, S. Yamauchi, R. Saito and T. Kyotani, Nano Lett., 2005, 5, 2465-2469.

5 Q. Yang, W. Xu, A. Tomita and T. Kyotani, J. Am. Chem. Soc., 2005, 127, 8956-8957.

6 P. He, X.-Y. Yu and X. W. Lou, Angew. Chem., 2017, 129, 39553958.

7 T.-W. Kim, I.-S. Park and R. Ryoo, Angew. Chem., 2003, 115, 4511-4515.

8 M.-M. Titirici, R. J. White, N. Brun, V. L. Budarin, D. S. Su, F. del Monte, J. H. Clark and M. J. MacLachlan, Chem. Soc. Rev., 2015, 44, 250-290.

9 H. Furukawa, K. E. Cordova, M. O'Keeffe and O. M. Yaghi, Science, 2013, 341, 1230444.

10 N. Stock and S. Biswas, Chem. Rev., 2012, 112, 933-969.

11 H. Wang, Q.-L. Zhu, R. Zou and Q. Xu, Chem, 2017, 2, 52-80. 
12 W. Shang, X. Kang, H. Ning, J. Zhang, X. Zhang, Z. Wu, G. Mo, X. Xing and B. Han, Langmuir, 2013, 29, 13168-13174.

13 Y.-S. Li, H. Bux, A. Feldhoff, G.-L. Li, W.-S. Yang and J. Caro, Adv. Mater., 2010, 22, 3322-3326.

14 S. Dang, Q.-L. Zhu and Q. Xu, Nat. Rev. Mater., 2017, 3, 17075.

15 B. Liu, H. Shioyama, T. Akita and Q. Xu, J. Am. Chem. Soc., 2008, 130, 5390-5391.

16 P. Zhang, F. Sun, Z. Xiang, Z. Shen, J. Yun and D. Cao, Energy Environ. Sci., 2014, 7, 442-450.

17 L. Wang, F. Ke and J. Zhu, Dalton Trans., 2016, 45, 45414547.

18 G. Zhao, Y. Fang, W. Dai and N. Ma, RSC Adv., 2017, 7, 21649-21654.

19 B. Tang, W.-C. Song, E.-C. Yang and X.-J. Zhao, RSC Adv., 2017, 7, 1531-1539.

20 W. Chaikittisilp, N. L. Torad, C. Li, M. Imura, N. Suzuki, S. Ishihara, K. Ariga and Y. Yamauchi, Chem.-Eur. J., 2014, 20, 4217-4221.

21 R. R. Salunkhe, C. Young, J. Tang, T. Takei, Y. Ide, N. Kobayashi and Y. Yamauchi, Chem. Commun., 2016, 52, 4764-4767.

22 H. J. Lee, W. Cho and M. Oh, Chem. Commun., 2012, 48, 221223.

23 J. Tang and Y. Yamauchi, Nat. Chem., 2016, 8, 638.
24 W. Zhang, X. Jiang, Y. Zhao, A. Carné-Sánchez, V. Malgras, J. Kim, J. H. Kim, S. Wang, J. Liu, J.-S. Jiang, Y. Yamauchi and M. Hu, Chem. Sci., 2017, 8, 3538-3546.

25 X.-H. Liu, W.-L. Hu, W.-J. Jiang, Y.-W. Yang, S. Niu, B. Sun, J. $\mathrm{Wu}$ and J.-S. Hu, ACS Appl. Mater. Interfaces, 2017, 9, 28473-28477.

26 M. Hmadeh, Z. Lu, Z. Liu, F. Gándara, H. Furukawa, S. Wan, V. Augustyn, R. Chang, L. Liao, F. Zhou, E. Perre, V. Ozolins, K. Suenaga, X. Duan, B. Dunn, Y. Yamamto, O. Terasaki and O. M. Yaghi, Chem. Mater., 2012, 24, 3511-3513.

27 R. R. Salunkhe, Y. V. Kaneti, J. Kim, J. H. Kim and Y. Yamauchi, Acc. Chem. Res., 2016, 49, 2796-2806.

28 M.-S. Wu and W.-H. Hsu, J. Power Sources, 2015, 274, 10551062.

29 K. László, E. Tombácz and K. Josepovits, Carbon, 2001, 39, 1217-1228.

30 B. Sun, J. Liu, A. Cao, W. Song and D. Wang, Chem. Commun., 2017, 53, 6303-6306.

31 D. Sheberla, J. C. Bachman, J. S. Elias, C. J. Sun, Y. Shao-Horn and M. Dinca, Nat. Mater., 2017, 16, 220-224.

32 Y. Jiao, J. Pei, D. Chen, C. Yan, Y. Hu, Q. Zhang and G. Chen, J. Mater. Chem. A, 2017, 5, 1094-1102.

33 Z. Weng, Y. Su, D.-W. Wang, F. Li, J. Du and H.-M. Cheng, Adv. Energy Mater., 2011, 1, 917-922. 